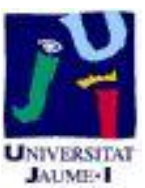

Título artículo / Títol article: A new window to understanding individual differences in reward sensitivity from attentional networks

Autores / Autors

Costumero Ramos, Victor ; Barrós Loscertales, Alfonso Roberto ; Bustamante, Juan Carlos ; Fuentes Claramonte, Paola ; Rosell Negre, Patricia ; Ventura Campos, Noelia ; Ávila Rivera, César

Revista:

Brain Structure and Function, 2014

Versión / Versió:

Preprint del autor

Cita bibliográfica / Cita

COSTUMERO, V., et al. A new window to understanding bibliogràfica (ISO 690): individual differences in reward sensitivity from attentional networks. Brain Structure and Function, 2014, vol. 220, no 3, p. 1807-1821.

url Repositori UJI:

http://hdl.handle.net/10234/125348 


\title{
A New Window to Understanding Individual Differences in Reward Sensitivity from Attentional Networks
}

\author{
Costumero $\mathrm{V}^{1}$, Barrós-Loscertales $\mathrm{A}^{1}$, Bustamante JC ${ }^{1}$, Fuentes $\mathrm{P}^{1}$, Rosell-Negre $\mathrm{P}^{1}$, \\ Ventura-Campos $\mathrm{N}^{1}$ and Ávila $\mathrm{C}^{1}$ \\ ${ }^{1}$ Departamento de Psicología Básica, Clínica y Psicobiología, Universitat Jaume I. Av. \\ de Vicent Sos Baynat, s/n 12071 Castelló de la Plana, (Spain)
}

Corresponding autor: Víctor Costumero Ramos, Av. de Vicent Sos Baynat, s/n 12071 Castelló de la Plana, (Spain). Tel. 34964387655 vcostume@uji.es.

\begin{abstract}
:
Existing evidence suggests that the presence of reward cues modifies the activity in attentional networks; however, the nature of these influences remains poorly understood. Here, we performed independent component analysis (ICA) in two fMRI datasets corresponding to two incentive delay tasks, which compared the response to reward (money and erotic pictures) and neutral cues, and yielded activations in the ventral striatum using a General Linear Model approach. Across both experiments, ICA revealed that both the right frontoparietal network and default mode network time courses were positively and negatively modulated by reward cues, respectively. Moreover, this dual neural response pattern was enhanced in individuals with strong reward sensitivity. Therefore, ICA may be a complementary tool to investigate the relevant role of attentional networks on reward processing, and to investigate reward sensitivity in normal and pathological populations.
\end{abstract}

Keywords: Functional connectivity; Independent Component Analysis; Reward; Sensitivity to reward; Frontoparietal network; Default mode network. 


\section{Introduction:}

Emotion and attention represent fundamental psychological processes that influence perception, action and conscious experience. Humans confront a myriad of simultaneous competing stimuli with limited processing capacity. The brain must meet the challenge of selecting only those stimuli most relevant for ongoing behavior and survival. In this sense, presence of reward cues is widely thought to modulate the salience of behavioral goals and to influence attention and behavioral control in relation to goal pursuit and completion (Kruglanski et al. 2002; Pessoa 2009). Nevertheless, little is known about the neurobiological mechanisms by which appetitive motivation influences attentional processing and cognitive control.

Respective neurobiological models of reward processing and attentional control propose that these functions are associated with different brain areas. Reward processing has been associated with a brain system that involves the core structures of the mesocorticolimbic dopamine system, including the midbrain, striatum, orbitofrontal cortex and amygdala (see Haber and Knutson 2010 for a review). Otherwise, attentional processing has been related with the activity of the different brain networks comprising primarily the parietal and frontal areas (Petersen and Posner 2012; Corbetta and Shulman 2002). In order to study how these neurobiological systems interact, in the present study we investigated whether reward cues capture more attention than neutral cues; that is, if attentional networks are involved in anticipation of reward. Different meta-analysis reviews on the brain areas involved in reward processing have provided mixed support as to the involvement of fronto-parietal areas in the processing of reward cues (see Knutson and Greer 2008; Liu et al. 2011; Diekhof et al. 2012; Sescousse et al. 2013). While all these studies have indicated a strong involvement of the reward brain areas in the processing of reward cues (i.e., ventral striatum and OFC), only one review has demonstrated the involvement of the left inferior parietal cortex and the left dorsolateral prefrontal cortex. Nonetheless, these results were not replicated using an alternative meta-analytic method (see Liu et al. 2011). Such scarce evidence contrasts with the psychophysiological studies showing enhanced P300 when processing reward cues and outcomes (Parvaz et al. 2012; van Lankveld and Smulders 2008; Yeung and Sanfey 2004), and with functional connectivity studies showing increased coupling between the parietal and striatal areas in the presence of reward cues (Padmala and Pessoa 2011). Furthermore, fMRI studies using attentional paradigms have revealed the greater involvement of the fronto-parietal areas when processing rewarding stimuli that 
compete with other stimuli (Small et al. 2005; Locke and Braver 2008; Mohanty et al. 2008; Engelmann et al. 2009; Ivanov et al. 2011).

In summary, fMRI studies on reward processing have not demonstrated consistent activity in attentional networks in the presence of reward cues, and have only showed the participation of these networks when using complex attentional tasks. However, these phenomena may be more intertwined than they appeared at first glance. One possibility of these results is that most reward-related studies rely on general linear model (GLM) analysis which focuses on investigating the functional specialization of discrete brain regions, while attentional processing is thought to be mediated by the interactions of different functional networks. An independent component analysis (ICA) applied to fMRI data is a functional connectivity method that allows the investigation of how the activity of functional networks is modulated by task conditions. The ICA is a data-driven approach which assumes that fMRI data are linear mixtures of independent source signals, and it attempts to extract maximally independent signals and their mixing coefficients. The driving principle behind ICA is that these independent source signals represent coherent groupings of MRI activations, often referred to as component maps, which implies the representation of a functionally connected network. Unlike conventional GLM analysis, ICA may serve to reveal the hidden factors that underlie sets of variables, measurements or signals, allowing the study of the time courses of a component (i.e., a functional network) separated from the signals of the others components (i.e., others networks and/or artifacts). Thus, the analysis of task-related modulations in the time courses of the functional networks generated by ICA can provide new insights into the brain's functional organization that are not observed in conventional GLM analysis (Xu et al. 2013).

A set of functional networks widely replicated across ICA studies (Allen et al. 2011; Biswal et al. 2010; Segall et al. 2012) has been associated with attentional processing. This set of networks includes the dorsal attentional network (DAN), related with goal-directed selection for stimuli and responses (Corbetta and Shulman 2002); the fronto-parietal network (FPN), related with the detection of behaviorally relevant stimuli and cognitive control (Corbetta and Shulman 2002; Vincent et al. 2008); and the default mode network (DMN), related with internal cognition (Raichle et al. 2001; Greicius et al. 2003). Furthermore, current neurobiological models emphasize the importance of interactions between these networks for cognitive control. Specifically, it has been proposed that the DAN and DMN increase their activity and couple with the 
FPN depending on how the attention toward relevant external or internal stimuli, respectively, is oriented (Spreng et al. 2012).

The objective of the present study was to investigate the participation of attentional networks in the processing of simple reward cues, and to investigate how this process is modulated by individual differences in reward sensitivity. To this end, we performed an fMRI experiment using the monetary incentive delay task (Knutson et al. 2001), and we carried out an ICA to identify the functional networks that respond to anticipation to reward and correlate with reward sensitivity. Furthermore, we performed similar analyses in a second, independent fMRI experiment in which the participants performed an adaptation of the incentive delay task using erotic images as rewarding stimuli. With this second experiment, we expected to generalize the results of Experiment 1 using a different kind of reward. Seeing that our reward cues were external in both cases, we hypothesized a positive modulation of the networks involved in externally oriented attention (DAN and FPN) and a negative DMN modulation during the processing of reward cues. A second interesting point of this research lies in the study of individual differences in reward sensitivity. While studies using rewardrelated tasks have shown modulation of reward sensitivity in the brain areas associated with the reward system (Beaver et al. 2006; Carter et al. 2009; Hahn et al. 2009; BarrósLoscertales et al. 2010; Costumero et al. 2013), studies focused on the interactions between motivation and cognitive control have demonstrated modulatory effects of reward sensitivity on the activity and connectivity of the parietal and frontal areas (Locke and Braver 2008; Engelmann et al. 2009; Padmala and Pessoa 2011). Consequently, we hypothesized that the expected modulation in the DAN, FPN and DMN in the presence of reward cues would be more prominent in those individuals displaying stronger reward sensitivity.

\section{Materials and methods:}

\section{Experiment 1}

\section{Participants}

Forty-one male undergraduates (mean age $=23.3, \mathrm{SD}=4.1$; mean years of education $=13.7, \mathrm{SD}=2.2$ ) participated in this fMRI study. Participants were physically and psychologically healthy with no history of mental disorders, head trauma, or drug 
abuse. Participants were informed of the nature of the research and signed written informed consent prior to participation. The study was approved by the Ethical Committee of the Universitat Jaume I (Spain). All study procedures conformed with the Code of Ethics of the World Medical Association (Declaration of Helsinki, 2013).

\section{Personality assessment}

The Sensitivity to Reward (SR) Scale from the Sensitivity to Punishment and Sensitivity to Reward Questionnaire (SPSRQ; Torrubia et al. 2001) was used as a measure of the reward sensitivity trait. Participants' SR mean score $(\mathrm{M}=11.6, \mathrm{SD}=4.41$, range: 3-21) was similar to those obtained in previous studies (Caseras et al. 2003; Barrós-Loscertales et al. 2006). The SPSRQ has been translated into 15 languages and is widely used to assess reward sensitivity in adults (Torrubia et al. 2001) and children (Luman et al. 2012). The SR scale has good content validity and strongly correlates with other measures of reward sensitivity, such as reward responsiveness, drive, fun seeking, novelty seeking, and impulsivity scales (Caseras et al. 2003).

\section{Experimental Design and Stimuli}

This experiment was designed to study the relationship between individual differences in the reward sensitivity personality trait and brain activity during anticipation to possible rewards and punishments. The task was an adaptation of the monetary incentive delay task described by Knutson et al. (2001) and included all the high and low reward and punishment conditions (see Online Resource 1). Before entering the scanner, all the participants were given instructions on the task and completed a practice session. The practice session was thought to minimize later learning effects and provided an estimate of each individual's reaction time (RT) to standardize task difficulty in the scanner. For each participant, the median RT of correct trials during the practice session was implemented as a cut-off RT in the main experiment. All the participants were initially paid 20 euros for their participation. At the end of the experiment, participants received an individually adjusted bonus depending on their performance in the experimental task.

Inside the scanner, participants performed two 8-minute runs of the monetary incentive delay task. Each run consisted of 60 trials for 120 trials in all. There were four kinds of events defined by a high reward, low reward, high punishment and low punishment cue. Each trial consisted of one of those cues, which was presented for 500 
ms. It was followed by a black screen of variable duration (2000-2250 ms) and then by a white target square that appeared for $100 \mathrm{~ms}$ to which participants had to respond by pressing a response button as quickly as possible. After the participant responded, a black screen with a variable duration of 2000-4000 ms appeared, followed by a feedback screen (1500 ms duration) that notified the participants whether they had won or lost money during that trial and indicated their cumulative total at that point. As previously noted, each event was defined by the initial appearance of a different cue: a circle with two horizontal lines indicating the possibility of winning 3 euros (a high reward cue; $n=24)$; a circle with one horizontal line indicating the possibility of winning 0.20 euros (a low reward cue; $n=24$ ); a square with two horizontal lines indicating the chance of avoiding losing 3 euros (a high punishment cue; $n=24$ ); a square with one horizontal line indicating the chance of avoiding losing 0.20 euros (a low punishment cue; $n=24)$. A triangle $(n=24)$ was the cue for non-incentive trials in which the participants neither won nor lost money. The participants had to respond after each incentive signal, but they did not respond to non-incentive signals since they were not followed by a target stimulus (a white square).

Trial types were pseudo-randomly ordered within each run. The intertrial interval was randomized between $2000 \mathrm{~ms}$ and $4000 \mathrm{~ms}$. Participants were instructed to respond as quickly as possible to target stimuli in order to achieve rewards or to avoid punishments. The task was programmed and presented using the Presentation software (Neurobehavioral Systems, Inc., Albany, USA). Visual stimuli were displayed in the scanner using Visuastim goggles (Resonance Technology, Inc., Northridge, USA). Stimulus presentation was synchronized with scanner acquisition using SyncBox (Nordic NeuroLab, Bergen, Norway) and behavioral task performance was recorded with a ResponseGrip (Nordic NeuroLab, Bergen, Norway). RT and the percentage of hits (successful responses to obtain rewards or to avoid punishments) were recorded as behavioral data.

\section{fMRI acquisition}

Image acquisition was performed using a 1.5T Siemens Avanto MRI scanner (Siemens, Erlangen, Germany). Functional images were acquired using a T2*-weighted echo-planar imaging sequence $(\mathrm{TR} / \mathrm{TE}=2000 / 30 \mathrm{~ms}$, matrix $=64$ x 64 x 30, flip angle $=90^{\circ}$, number of volumes $=502$ ). Thirty 3.5 -mm-thick slices centered parallel to the hippocampi were axially acquired with a 0.5 -mm interslice gap. 


\section{Image Preprocessing}

Image processing was carried out using SPM8 (Statistical Parametric Mapping 8; The Wellcome Department of Cognitive Neurology, London, UK). Preprocessing of the functional scans included noise filtering using an Art Repair toolbox (http://cibsr.stanford.edu/tools/human-brain-project/artrepair-software.html) to repair slice artifacts through interpolation, slice-timing correction, realignment to correct for motion-related artifacts, spatial normalization into the standard Montreal Neurological Institute space using SPM8's EPI template (voxel size $3 \mathrm{~mm}^{3}$ ) and smoothing with fullwidth at a half maximum (FWHM) of the Gaussian kernel ( $8 \mathrm{~mm})$.

\section{GLM analysis:}

The GLM analysis (Friston et al. 1995) was performed with SPM8 to study brain activity in response to anticipatory cues. In the first-level analyses, a statistical model was computed for each participant. The GLM design matrix included separate regressors for each anticipatory cue, feedbacks and targets by applying a canonical hemodynamic response function and its time derivative. In addition, the six motion correction parameters from each participant were included in the model as 'nuisance' variables. The fMRI time series data were high pass-filtered with a cut-off frequency of $1 / 128 \mathrm{~Hz}$ to eliminate low-frequency components. Finally, statistical contrast images were generated by comparing reward (high and low) and non-incentive cues, as well as punishment (high and low) and non-incentive cues, to obtain brain activation for anticipatory periods.

The second-level analyses consisted in two one-sample t-tests using the contrast images from the first-level analyses. Region of interest (ROI) analyses were carried out to study brain activity for each contrast in the bilateral ventral striatum (VS), an area that has been highly related with reward anticipation (Knutson et al. 2001, 2005). The VS ROIs were defined as a 6 -mm-radius sphere at the $[ \pm 10,8,-4]$ MNI coordinates (based on Barrós-Loscertales et al. 2006). The statistical threshold was defined using small volume correction (SVC) at $\mathrm{p}<0.05$, FWE-corrected at the voxel level. Furthermore, we extracted the mean ROI activity for each contrast by averaging the beta-weights from those active voxels within the ROI. Then, the mean activity of the ROIs was correlated with the RTs and SR-scores in order to study the behavioral and personality relations with brain activity. 


\section{$\underline{\text { Independent Component Analysis }}$}

Group ICA was performed to obtain the functional brain networks underlying the fMRI data. ICA is a statistical method used to discover hidden factors from a set of measurements or observed data so that sources are maximally independent (see Calhoun et al. 2009 for a review). When applied to fMRI data, spatial ICA identifies temporally coherent networks which are spatially maximally independent. The main advantage of ICA is that it does not require a priori models of brain activity or connectivity to generate functional networks because it is a data-driven approach.

Group ICA was done using Gift toolbox (v1.3i, http://icatb.sourceforge.net). The optimal number of independent components (ICs) was 20, which were calculated using the minimum description length (MDL) criteria (Li et al. 2007). Principal component analysis (PCA) was used to reduce data dimensionality following a two-step data reduction approach (Calhoun et al. 2001). Then, ICA decomposition was performed with an Infomax algorithm (Bell and Sejnowski 1995) to reach the final number of 20 ICs found with the MDL criteria. Twenty ICA iterations were performed by ICASSO (Himberg et al. 2004) to ensure the stability of the estimated ICs (see Online Resource 2). Then, individual IC maps and time courses were computed using backreconstruction based on the aggregate components of the ICA and the results from the data reduction step (Erhardt et al. 2011). Finally, individual ICs were scaled to z-scores.

The spatial maps generated by ICA were averaged across runs and one-sample ttests at the second-level analyses were performed with SPM8 (at $\mathrm{p}<1 \times 10^{-12}$ FDRcorrected; $\mathrm{k}=30$ ) to determine the brain regions that significantly relate with each IC time courses for the whole group. This analysis provides a map of functionally connected brain regions belonging to each IC.

Following previous studies (Kim et al. 2009; Ye et al. 2012; Juárez et al. 2012), we performed GLM analyses on the IC time courses to analyze the engagement of functional networks under tasks conditions. Thus, the regression analysis was performed in each IC time course using the estimated GLM design matrix. This analysis yields a set of beta-weights representing the modulation of the ICs time courses by the GLM regressors in relation to the baseline. The beta-weights for each condition were averaged across runs. Furthermore, the beta-weights for reward (high and low) and punishment (high and low) conditions were also averaged in order to acquire a whole 
measure of reward and punishment anticipation as this facilities comparability with Experiment 2. These beta-weights were then used to perform the second-level analyses.

As ICA constitute a data-driven approach and since some ICs may represent artifacts or brain networks that do not relate to the experimental conditions, we used 3step IC selection criteria based on previous studies (Kim et al. 2009; Sambataro et al. 2010; Ye et al. 2012). Thus, the ICs of interest were selected in three consecutive stages: 1) those ICs whose ICASSO-calculated coefficient of stability was lower than 0.9 were considered unstable and were removed for further analyses; 2) the ICs were correlated with prior probabilistic maps of gray matter, white matter and cerebral spinal fluid (CSF), provided by the MNI templates of SPM8, so that those ICs with a spatial correlation higher than $r^{2}=0.02$ with white matter, greater than $r^{2}=0.05$ with CSF or a lower correlation with gray matter than the correlation with white matter or CFS was not considered to be primarily located within grey matter and removed; 3) one-way repeated measures ANOVA were performed with the beta-weights for each anticipatory condition to determine which IC showed differential involvement during the anticipatory period for the whole group, and the ICs that did not show significant differences at the $\mathrm{p}<0.05$ FDR-corrected level were considered to not be task-related and were removed for subsequent analyses.

After identifying the ICs of interests relating with the task using ANOVA, post hoc analyses were performed with these ICs to study how time courses were modulated by the different task conditions $(\mathrm{p}<0.05$, corrected). Furthermore, correlation analyses were done using the behavioral and personality variables to study their relationship with the modulation of the functional connected brain networks by task conditions. Thus, the beta-weights for each anticipatory cue of those ICs of interest correlated with both the SR scores and the mean RT.

Experiment 2

\section{$\underline{\text { Participants }}$}

Thirty heterosexual men (mean age $=23.7, \mathrm{SD}=3$; mean years of education $=$ 13.9, $\mathrm{SD}=2.4$ ) took part in this study. None of the participants included in the study reported a history of DSM-IV Axis I or II disorders, severe medical illnesses or neurological illnesses, history of head injury with loss of consciousness, or current use of psychoactive medications. Participants were informed of the nature of the research, 
provided written informed consent prior to participating in this study and were paid $€ 30$ for their participation. The study was approved by the Human Subjects Committee of the Universitat Jaume I of Castellón (Spain). All study procedures conformed with the Code of Ethics of the World Medical Association (Declaration of Helsinki, 2013).

\section{$\underline{\text { Personality assessment }}$}

As in Experiment 1, we used the SR scale from the SPSRQ. The participants' mean SR score was 11.5 ( $\mathrm{SD}=5.3$ range: 3-20), similar to Experiment 1 and previous studies (Caseras et al. 2003; Barrós-Loscertales et al. 2006).

\section{Experimental design and stimuli}

In order to study the role of the reward sensitivity personality trait in anticipation to non-monetary rewarding stimuli we performed an incentive delay task including erotic and neutral pictures as an outcome (See Online Resource 1). The instrumental task began with a discriminative cue presented for $500 \mathrm{~ms}$ which signalled the trial type. A white square (target) appeared after the cue and remained for $100 \mathrm{~ms}$ in a random interval lasting between 2 and 2.25 seconds. Whenever the participants responded, a picture was presented for 1 second after a randomizing interval (6-10 seconds). The participants were asked to make a response when they saw the target stimuli. When the participants did not respond within a 2-second temporal interval (response window) after the target stimuli presentation, a "\#” symbol appeared for 1 second, indicating that this trial had not been properly performed. Four experimental trial types were included in this experiment in order to manipulate the motivational value of the anticipatory cue and the motor response anticipation effects. In the continuous reward trials $(n=32)$, " $X$ " indicated that whenever the participants responded to the target stimulus, an erotic picture would be presented. In the partial reward trials $(n=32)$, “?” indicated that the participants had to respond quickly to view an erotic picture, otherwise a neutral picture would be presented. Thus as in the continuous reward trial, this condition involved anticipatory responses to reward stimuli, but in this case, the outcome depended on the participants' RT. The task difficulty for these trials was individualized for each subject based on RT, and was updated during the task depending of on-going execution, thus ensuring at least about $60 \%$ of accuracy. In the neutral trials, a "triangle" (n=32) indicated that participants would be presented with a neutral picture after their response. Hence, this condition involves action preparation to respond to target stimuli correctly, 
but without emotional contingences. Finally in the control trials, the participants passively saw a "circle" $(\mathrm{n}=32)$ followed by a neutral picture without the target stimuli being presented. Therefore, this last condition did not involve motivational effects neither motor preparation, allowing us study the modulation of attentional networks by action preparation without motivational contingences, since previous studies have shown a main effect of motor preparation on the activity of key regions within the reward system (Guitart-Massip et al. 2011). The task was divided into four runs. Each run consisted of 32 trials with 128 trials in all. Trial types were pseudo-randomly ordered within each run. The inter-trial interval was randomized between 6 and 10 seconds. The erotic pictures set included photographs of couples and undressed adult women, whereas the neutral pictures set included house-hold items and scenes of daily life. The resolution image was $800 \times 600$ pixels and no picture was shown more than once. Before entering the scanner, all the participants were given instructions about the task and completed a practice session to minimize later learning effects. After the scan session, the subjects valued all the pictures on both the valence and arousal dimensions (on a scale of 1-9). The task programming software and stimulus presentation tools were the same as in Experiment 1. RT and the percentage of hits (successful responses in partial reward trials) were recorded as behavioral data.

\section{$\underline{\text { FMRI Acquisition }}$}

Image acquisition was performed using a $1.5 \mathrm{~T}$ Siemens Avanto MRI scanner (Siemens, Erlangen, Germany). Functional images were acquired using a T2*-weighted echo-planar imaging sequence $($ Slices per volume $=30, \mathrm{TR} / \mathrm{TE}=2500 / 48 \mathrm{~ms}$, matrix $=$ $64 \times 64 \times 30$, flip angle $=90^{\circ}$, number of volumes $=840$ ). Thirty 3.5-mm-thick slices centered to AC-PC were axially acquired with a $0.3-\mathrm{mm}$ interslice gap.

\section{$\underline{\text { Image Preprocessing }}$}

The preprocessing implemented in the fMRI images for this experiment was the same as in Experiment 1.

\section{GLM analysis:}

The GLM analyses for this experiment were similar to Experiment 1. After performing the GLM design matrix, statistical contrast images were generated by separately comparing the continuous and partial trials with neutral and control trials. 
One-sample t-test was done in the second-level analysis for each generated contrast and ROI analyses were carried out in the VS using SVC at p<0.05, FWE corrected at the voxel level. In addition, the mean activity of the ROIs was correlated with their respective mean $\mathrm{RT}$ and SR-scores in order to study the behavioral and personality relations with brain activity.

\section{Independent Component Analysis}

The ICA performed for this experiment was similar to those implemented in Experiment 1 , the only difference being that the optimal number of ICs determined by the MDL criteria for this experiment was 28.

\section{Results:}

Experiment 1

Behavioral results:

The means and standard deviations for RT and hits are presented in Table 1. Paired t-tests were performed to study the differences in RT and hits between conditions. Despite the differences between conditions being small, the results reveal that participants responded significantly faster $\left(\mathrm{t}_{40}=-2.4 ; \mathrm{p}=0.02\right)$ and more successfully $\left(\mathrm{t}_{40}=3.9 ; \mathrm{p}<0.001\right)$ for reward cues than for punishment cues.

\section{$\underline{\text { GLM results }}$}

As expected, ROI analyses (See Fig. 1) showed increased bilateral VS activity while reward cues were presented as compared to the non-incentive cues (right: MNI peak maximum $=6,8,1, \mathrm{Z}$-score $=3.65, \mathrm{k}=9$; left: $\mathrm{MNI}$ peak maximum $=-6,8,1, \mathrm{Z}$ score $=3.07, \mathrm{k}=4)$. Furthermore, increased right VS activity was noted during the presentation of the punishment cues in comparison to the non-incentive cues (MNI peak maximum $=6,8,1, \mathrm{Z}$-score $=3.13, \mathrm{k}=4)$. These results are in consonance with previous reports showing increased VS activity while processing both the reward and punishment anticipatory cues (Carter et al. 2009). The whole brain voxel-wise results for each contrast are summarized in Online Resource 3. 
Neither the associations between the activity of the ROIs and the SR-scores in the correlation analyses nor the correlations between the activity of the ROIs and RTs were significant.

\section{$\underline{\text { ICA results }}$}

Four ICs (C4, C5, C9 and C10) passed the selection criteria and were, therefore, selected as ICs of interest. Each IC was defined based on the similarities between the ICs spatial maps and the networks shown in previous resting state studies using bigger samples (Allen et al. 2011; Segall et al. 2012). Thus, the four ICs of interest were identified as the DMN (C4), the left FPN (C5), the right FPN (C9) and the frontal network (C10). No component resembling the DAN was obtained in this experiment. Table 2 summarizes the brain areas belonging to the spatial map of each IC of interest.

The one-way repeated measures ANOVA showed how each IC of interest was modulated by anticipatory conditions (see Fig. 2 and Fig. 3). The DMN exhibited a negative modulation for the reward and punishment cues in comparison to the nonincentive cues. Furthermore, the left FPN displayed a greater negative modulation for the reward cues than for the non-incentive cues, while the right FPN showed a positive modulation for the reward cues in comparison to the punishment and non-incentive cues. No differences between punishment and the non-incentive cues were found for both the right and left FPN, suggesting specific FPN engagement during reward anticipation. Finally, the frontal network displayed lower negative modulation for both the reward and punishment cues than for the non-incentive cues.

Correlation analyses between the SR scores and beta-weights for each anticipatory condition were done to study the relationship between reward sensitivity and functional networks (Table 3). This analysis showed that the SR scores correlated negatively with the DMN during the anticipation of both monetary rewards and punishments. In addition, the SR scores correlated positively with the right FPN during the anticipation of both monetary rewards and punishments.

We also performed correlation analyses to investigate how the modulation of the functional networks by the tasks conditions was related with the mean RTs. Nevertheless, no significant correlations between the RTs and ICs beta-weights were found under any anticipatory condition. 
Experiment 2

Behavioral results:

The means and standard deviations for RT, hits and subjective ratings are presented in Table 1. A one-way repeated measure ANOVA was performed using the RTs for each incentive condition to study differences in performance. Significant differences were obtained between the RTs' incentive cues $\left(\mathrm{F}_{(1.5,45)}=9.7 ; \mathrm{p}=0.001\right)$ showing that the participants were faster for partial reward than for continuous reward and neutral cues.

Two paired t-tests were run according to the participants' image ratings to study the subjective reward stimuli value. The analyses revealed that the erotic picture set was significantly rated as more pleasant $\left(\mathrm{t}_{29}=9.2 ; \mathrm{p}<0.001\right)$ and arousing $\left(\mathrm{t}_{29}=11.1 ; \mathrm{p}<0.001\right)$ than the neutral picture set, thus confirming that erotic pictures were subjectively positive for participants.

\section{$\underline{\text { GLM results }}$}

The ROI analyses in this experiment (See Fig. 1) showed higher bilateral VS activity during the presentation of partial reward as compared to the neutral cues (right: MNI peak maximum $=15,8,-5, \mathrm{Z}$-score $=4.31, \mathrm{k}=38$; left: $\mathrm{MNI}$ peak maximum $=-9$, $5,-2, \mathrm{Z}$-score $=4.22, \mathrm{k}=33$ ) and to the control cues (right: $\mathrm{MNI}$ peak maximum $=9$, $11,-2, \mathrm{Z}$-score $=3.76, \mathrm{k}=12$; left: $\mathrm{MNI}$ peak maximum $=-9,5,-5, \mathrm{Z}$-score $=3.65, \mathrm{k}=$ 22). Furthermore, increased activity in the left VS was seen during continuous reward cues as compared to both the neutral (MNI peak maximum $=-9,8,1, \mathrm{Z}$-score $=3.4, \mathrm{k}=$ 17 ) and control (MNI peak maximum $=-9,8,-5, \mathrm{Z}$-score $=3.16, \mathrm{k}=12)$ cues. These results generalize to sexual rewards the findings showing VS involvement in anticipation to monetary rewards (Knutson et al. 2001). The whole brain voxel-wise results for each contrast are summarized in Online Resource 3.

Furthermore, the analyses investigating the association between the mean activity of the ROIs and RTs revealed that under the partial reward condition, right VS activity negatively correlated with the mean RTs $(r=-0.47 ; p=0.009)$. Thus, those participants exhibiting stronger VS activity displayed faster RTs under the partial reward condition. No association between the activity of the ROIs and the personality assessments was observed in any ROI. 


\section{$\underline{\text { ICA results }}$}

For this experiment, four ICs (C3, C7, C19 and C26) passed the selection criteria and were, therefore, selected as ICs of interest. Similarly to Experiment 1, each IC was defined based on its similarities with the networks shown in previous studies (Allen et al. 2011; Segall et al. 2012). Thus, the four ICs of interest shown in this experiment were identified as the left sensory motor network (C3), the right FPN (C7), the DMN (C19) and the cerebellum (C26). In this experiment, the DAN (C23) did not pass the selection criteria given a greater spatial correlation than $r^{2}=0.02$ with white matter. The brain areas belonging to the spatial map of each IC of interest are summarized in Table 2.

The ANOVA results for this experiment (Fig. 2 and Fig. 3) showed that the left sensory motor network displayed a higher positive modulation for all the instrumental conditions (continuous reward, partial reward and neutral) than the control condition, suggesting the involvement of this network in preparation to movement. Additionally, the partial reward cues showed larger positive modulation than both the continuous and neutral cues, indicating that the requirement of fast responses had an effect on this network. The right FPN displayed a positive modulation for the reward conditions when compared with the control cues. The partial reward cues also showed a greater positive modulation than the neutral cues. Thus in general terms, the effects of the right FPN were modulated mainly by reward anticipation and the presence of reward cues requiring a faster response. The DMN exhibited a larger negative modulation for the instrumental conditions when compared to the control condition, which suggests its involvement in movement preparation. Additionally, the fact that the partial reward cues presented higher negative modulation than both the continuous reward and neutral cues once again hinted that the requirement of fast responses to obtain rewards modulates this network. Finally, the cerebellum showed an effect of movement preparation which was due to larger positive modulation for the instrumental cues than for the control cues. Despite the DAN not passing the selection criteria, its possible involvement in the task using ANOVA was checked. Nevertheless this component did not show taskrelated differences.

The Pearson's correlations between the SR scores and IC task-related modulation appear in Table 3, and reveal that the SR scores correlated negatively with DMN modulation upon the onset of the continuous and partial reward cues, but not while processing the neutral and control cues. Furthermore, the SR scores correlated 
positively with the right FPN modulation while processing all the conditions. Given this result, we ran additional partial correlations to ascertain if the reward or requirements of the motor response modulate the magnitude of the correlations. When regressed out for activity during the neutral condition, the correlation found between the SR scores and activity upon the onset of the continuous reward cues remained significant $(r=0.47, p=$ 0.009). The same correlation during partial reward showed a significant tendency $(\mathrm{r}=0.36, \mathrm{p}=0.055)$. When controlling for network modulations under the control condition, the SR scores correlated positively with the network modulation during partial $(\mathrm{r}=0.52, \mathrm{p}=0.003)$ and continuous $(\mathrm{r}=0.53, \mathrm{p}=0.003)$ reward. Thus, the presence of the reward cues seemed to contribute to modulate the activity in the right FPN.

Finally, the correlation analyses performed to study the relation between IC taskrelated modulation and the RTs gave a positive correlation between the DMN and the RTs under the partial reward condition $(\mathrm{r}=0.57 ; \mathrm{p}=0.001)$, indicating that the participants exhibiting stronger DMN deactivation also displayed faster RTs for this condition.

\section{Discussion:}

Across two different experiments, we used ICA to identify the anatomical components of the putative brain networks involved in processing reward cues based on their synchronous activation by filtering out the noise/artifactual components of the fMRI signal. We were also able to examine individual differences in the functioning of these networks in accordance with the reward sensitivity scores. Our results confirm our hypotheses about the involvement of two attentional networks in reward processing: the right FPN and the DMN. Specifically, we show that the presence of reward cues positively and negatively modulates the right FPN and the DMN, respectively. It is noteworthy that both effects were enhanced in individuals who obtained higher scores in reward sensitivity. These results reveal that a response to reward cues is not merely circumscribed to the "classical" reward brain areas, but to attentional networks that serve to better integrate all the information required to produce optimal decisions (Liu et al. 2011). The relevance of these data may also prove important for brain disorders associated with deficits in reward processing.

As far as we know, the present experiments are the first to investigate the relationship between attentional networks and the processing of reward cues using ICA. 
The results of both experiments consistently show that processing reward cues positively modulates right FPN activity and negatively modulates DMN activity, while there is no task effect for the DAN. Consistently with the DMN pattern of deactivation shown in other studies (Raichle and Snyder 2007), we demonstrated that the DMN is negatively modulated by reward cues in comparison to neutral ones. In Experiment 1, the DMN displayed negative modulation for both reward and punishment cues in comparison to the non-incentive cues. In Experiment 2, the results indicate that the DMN is negatively modulated by reward cues, especially when delivery of reward depends on a subject's performance. Furthermore under the partial reward condition, we found a positive correlation between DMN modulation and the RT, which indicates that the stronger the DMN deactivation, the faster the RT. The DMN has been associated with internal cognitive processes and it deactivates when attention is paid to external stimuli (Raichle et al. 2001; Greicius et al. 2003). Previous research has repeatedly shown that, during cognitive tasks, the higher the task demands, the stronger DMN deactivation is (Harrison et al. 2011). The present study also shows that the motivational stimulus value also modulates DMN deactivation independently of task demands since reward conditions are not cognitively harder than neutral ones. This result is consistent with a previous report which did ROI analyses to show that reward cues and cues anticipating high task demands deactivate specific DMN areas when compared to nonreward and low task demand cues (Krebs et al. 2012). Overall, the pattern of the DMN results is consistent with the proposal that the suppression of this network is important for goal-directed externally-oriented cognition (Anticevic et al. 2012; Spreng 2012).

The DAN is hypothesized to modulate externally directed attention by amplifying the saliency of the relevant cues of the environment in accordance with current action goals (Corbetta and Shulman 2002; Ptak and Schnider 2010). Nevertheless, this network did not prove relevant in this study. In Experiment 1, we were unable to identify the DAN in any component. In Experiment 2, we obtained the DAN in the ICA (C23), but this network did not showed differential involvement between task conditions. In other words, the presence of reward cues does not modulate DAN activity if compared with neutral cues. These results can be explained by the few spatial orienting, eye movement or visuospatial integration requirements involved in our tasks, especially when comparing reward and neutral cues. It is also feasible that this network focuses on controlling purely cognitive operations to guide spatial orienting in accordance with relevant stimuli and personal goals. The tasks used in Experiments 1 
and 2 presented only one stimulus per trial, and the lack of competition between stimuli may moderate the need for the top-down modulation associated with the DAN.

Finally, the right FPN was positively modulated by the reward cues when compared with the non-reward cues. In Experiment 1, we found that this network was more engaged in situations involving reward, whereas in Experiment 2, the presence of the reward cues and the requirement of fast responses to obtain reward positively modulated this network. It has been proposed that the right FPN is specialized in the detection of behaviorally relevant stimuli, particularly when they are salient or unexpected (Corbetta and Shulman 2002). In this sense, the results of the present study indicate that the activity of the network is not only related to the need to select between different stimuli in conflict monitoring, planning and reasoning (Kroger et al. 2002; van den Heuvel et al. 2003; Wager et al. 2004), but it also seems to exert an arousing effect when processing reward stimuli, and it probably participates in preparing the motor response.

The specific role of the right FPN has been recently depicted by considering the interaction of attentional networks and the characteristics of the task (Spreng et al. 2010, 2012). Vincent and colleagues (2008) noticed that the FPN is physically interposed between the DAN and the DMN, and these authors suggested that the FPN may flexibly couple to the DMN or the DAN, depending on the attentional demand of the task. Spreng and colleagues (2010) gave evidence about how both the DMN and DAN appear to compete for positive coupling with FPN. They reported increased DAN and FPN activity, but diminished DMN activity, when performing a visuospatial planning task, but found increased DMN and FPN activity and reduced DAN activity when performing an autobiographical planning task. These results led to the proposal that the FPN is coupled to not only the DMN during internal cognition, but also to the DAN during external cognition. The data obtained in the present study indicate that the DAN is not relevant in those tasks which require the processing of a single reward cue (i.e., not requiring selective attention), and only the right FPN and the DMN are positively and negatively involved, respectively. These results agree with previous studies which have shown a right lateralized pattern of activity in the frontoparietal areas present in the tasks involving vigilant attention, especially those requiring stimulus detection and not stimulus discrimination (see Langner and Eickhoff 2013). Vigilant attention involves an effortful process of endogenous maintaining appropriate attentional levels in unchallenging activities, and has been proposed to be a multicomponent, non unitary 
process that relies mainly on right hemisphere structures, including the FPN (Langner and Eickhoff 2013). In this context, the increased modulation of the right FPN by reward cues more than neutral ones may be interpreted as a function of intensity of attention triggered by each cue (Spitzer et al. 1988), which might reflect motivation induced by expected reward. All together, the results of this study are in line with the notion that the FPN probably couples its activity with relevant networks, such as the DMN, to endogenously focus attention on relevant stimuli by regulating sustained arousal (Chica et al. 2013).

This action of the attentional networks might be mediated by dopamine because, on the one hand, reward cues phasically increase dopamine in the brain (Wightman and Robinson 2002) and, on the other hand, activity in the right FPN is modulated by dopamine (Tan et al. 2007; Williams-Gray et al. 2007). The presence of reward cues phasically increases the firing of dopamine neurons in basal ganglia and the frontal cortex (Schultz et al. 1998; Stalnaker et al. 2012). Mesocorticolimbic dopamine has also been proposed to mediate not only increased activity and connectivity in the FPN (Tan et al. 2007; Fischer et al. 2010), but also the relationship of this network with other attentional networks (Dang et al. 2012). Likewise, dopamine was related to decreases in DMN activity (Nagano-Saito et al. 2009; Tomasi et al. 2011). Hence we may tentatively propose that the presence of reward cues leads to increases in right FPN activity and to decreases in DMN throughout dopamine.

This link between dopamine and activity in attention networks is indeed more relevant if we focus on the fact that the main effects obtained in this study are mainly driven by individual differences in reward sensitivity. In both experiments, we accomplished a modulation in the right FPN and the DMN, while processing reward cues related positively and negatively to the SR scores, respectively. Individual differences in reward sensitivity have been previously associated with structural abnormalities in the striatum (Barrós-Loscertales et al. 2006) and with dopamine levels (Pickering and Gray 2001). Previous studies have also shown that reward sensitivity is associated with stronger activity in the midbrain, striatum and OFC when processing reward cues (Hahn et al. 2009; Carter et al. 2009). For the first time, our ICA reveals that reward sensitivity is also linked to a distinct activity in attentional networks.

Previous studies have reported results that are consistent with our data. First, one interesting result as regards the right FPN was that reward sensitivity is associated with the modulation of this network under the non-rewarded conditions in Experiment 2, but 
not in Experiment 1. This result agrees with recent results that relate reward sensitivity to a stronger probability of adopting a proactive control mode in contexts with intermittent rewards (Jimura et al. 2010). Proactive control has been related with sustained and anticipatory activity in the right dorsolateral PFC, an area belonging to the right FPN. Thus, intermittent reward contexts with mixed reward and neutral trials can be associated with the adoption of a proactive mode by high reward sensitive subjects, which led them to the sustained and anticipatory maintenance of goal-relevant information throughout the task (Braver 2012). Based on the results of Experiment 1, we may tentatively propose that this effect should not be observed in mixed rewards and punishments contexts. The second related issue stems from diverse behavioral studies which show that individuals with stronger reward sensitivity possess an attentional system which is directed at seeking and effectively detecting relevant environmental stimuli by means of the conscious focalization of attention on locations or stimuli associated with reward (Derryberry and Reed 1994; Ávila 2001). These studies support the model depicted by Patterson and Newman (1993), which proposes that individuals with stronger reward sensitivity pay more attention to reward cues at the expense of ignoring other relevant stimuli such as punishment cues (Patterson et al. 1987; Ávila 2001). In other words, these individuals strongly focus their attention on reward cues, which reduces the probability of changing the reward-directed behavior by adverse consequences. The results of the present study agree with this theoretical model and show that when processing reward cues, individuals with stronger reward sensitivity present greater activity in the right FPN and more deactivation in the DMN than individuals with lower reward sensitivity. This notion supports the proposal that these individuals center their attention on reward cues more than individuals with lower reward sensitivity. Further studies should investigate the attentional processing of nonrewarded competing stimuli in those individuals who display stronger reward sensitivity. Third, psychophysiological research supports the present data. Parvaz et al. (2012) measured reward sensitivity from the amplitude of P300 to the expectation of different magnitudes of reward. Expectation of a high reward yielded a stronger P300 response than expectation of a non-reward, and this difference correlates with the gray matter volume of several prefrontal cortex areas. Consistently with the results of the present study, the above authors highlighted the importance of prefrontal integrity to modulate attentional responses to reward cues. Fourth, several neurocognitive models on individual differences applied to diverse fields such as psychopathology (Volkow et 
al. 2011), adolescence (Ernst et al. 2006) or personality (Pickering and Gray 2001), establish that attention modulates reward processing to a certain degree. The results of the present study offer a new procedure to investigate these effects and to test these models. In general terms, all these models propose that some frontoparietal areas modulate the action of the reward brain areas and that they help determine reward sensitivity and probability of approach. However, the specific effect of the DMN on these models is still not well-established.

Another point of interest in the present study is the comparison of the ICA and GLM results (Xu et al. 2013). Using the conventional GLM analyses, both the datasets employed in the present study have shown a consistent activation of the ventral striatum. However, conventional GLM analyses proved less specific to find consistent differences across studies in the areas included in the right FPN or the DMN networks. Besides, ICA showed the reverse pattern of results, with differences in attentional networks, but not in those networks involving classical "reward areas" such as the striatum. With these results, we can consider ICA to be a new, alternative way to investigate individual differences in reward sensitivity, which offer promising applications to psychiatric disorders (depression, psychopathy, ADHD, substance abuse, etc.) characterized by deficits in reward processing.

\section{Conclusions:}

Using ICA, we have shown that attentional networks are modulated by motivational cues across two reward-related tasks. Specifically, we demonstrate that reward cues positively modulate the right FPN and negatively modulate the DMN time course. We also show that the modulation in the right FPN and the DMN while processing reward cues relates positively and negatively to the SR scores, respectively. Finally, no differences were found in the modulation of DAN, suggesting that this network is less influenced by incentive motivation in tasks with fewer visuospatial requirements. The ICA procedure applied to reward processing opens a new window to investigate reward processing and individual differences in reward sensitivity.

\section{Acknowledgements:}

This research was supported by the Brainglot project of the CONSOLIDER-INGENIO 2010 Programme (CSD2007-00012). Also, the project was supported by grants PSI2010-20168 from MINECO, P1•1B2011-09 from Universitat Jaume I to CA, and 
grants 040/2011 from Spanish National Drug Strategy Ministerio de Sanidad y Consumo, GV/2012/042 from the GeneralitatValenciana and PSI2012-33054 from Ministerio de Economía y Competitividad to ABL.

The authors declare that they have no conflict of interest.

\section{References:}

Allen EA, Erhardt EB, Damaraju E, Gruner W, Segall JM, Silva RF, Havlicek M, Rachakonda S, Fries J, Kalyanam R, et al. (2011). A baseline for the multivariate comparison of resting-state networks. Front Syst Neurosci 5, 2.

Anticevic A, Cole MW, Murray JD, Corlett PR, Wang XJ and Krystal JH (2012). The role of default network deactivation in cognition and disease. Trends Cogn Sci 16, 584592.

Ávila C (2001). Distinguishing BIS-mediated and BAS-mediated disinhibition mechanisms: a comparison of disinhibition models of Gray $(1981,1987)$ and of Patterson and Newman (1993). J Pers Soc Psychol 80, 311-324.

Barrós-Loscertales A, Meseguer V, Sanjuán A, Belloch V, Parcet MA, Torrubia R and Ávila C (2006). Striatum gray matter reduction in males with an overactive behavioral activation system. Eur J Neurosci 24, 2071-2074.

Barrós-Loscertales A, Ventura-Campos N, Sanjuán-Tomás A, Belloch V, Parcet MA, Avila C (2010). Behavioral activation system modulation on brain activation during appetitive and aversive stimulus processing. Soc Cogn Affect Neurosci 5, 18-28.

Beaver JD, Lawrence AD, Van Ditzhuijzen J, Davis MH, Woods A and Calder AJ (2006). Individual differences in reward drive predict neural responses to images of food. J Neurosci 26, 5160-5166.

Bell AJ and Sejnowski TJ (1995). An information-maximization approach to blind separation and blind deconvolution. Neural Comput 7, 1129-1159.

Biswal BB, Mennes M, Zuo XN, Gohel S, Kelly C, Smith SM, Beckmann CF, Adelstein JS, Buckner RL, Colcombe S, et al. (2010). Toward discovery science of human brain function. Proc Natl Acad Sci U S A 107, 4734-4739.

Braver TS (2012). The variable nature of cognitive control: a dual mechanisms framework. Trends Cogn Sci 16, 106-113.

Calhoun VD, Adali T, Pearlson GD and Pekar JJ (2001). A method for making group inferences from functional MRI data using independent component analysis. Hum Brain Mapp 14, 140-151.

Calhoun VD, Liu J and Adali T (2009). A review of group ICA for fMRI data and ICA for joint inference of imaging, genetic, and ERP data. Neuroimage 45, S163-72. 
Carter RM, Macinnes JJ, Huettel SA and Adcock RA (2009). Activation in the VTA and nucleus accumbens increases in anticipation of both gains and losses. Front Behav Neurosci 3, 21.

Caseras X, Ávila C and Torrubia R (2003). The measurement of individual differences in Behavioural Inhibition and Behavioural Activation Systems: a comparison of personality scales. Pers Individ Dif 34, 999-1013.

Chica AB, Bartolomeo P and Lupiáñez J (2013). Two cognitive and neural systems for endogenous and exogenous spatial attention. Behav Brain Res 237, 107-23.

Corbetta M and Shulman GL (2002). Control of goal-directed and stimulus-driven attention in the brain. Nat Rev Neurosci 3, 201-215.

Costumero V, Barrós-Loscertales A, Bustamante JC, Ventura-Campos N, Fuentes P, Rosell-Negre P, Avila C, (2013). Reward Sensitivity Is Associated with Brain Activity during Erotic Stimulus Processing. PLoS One 8, e66940.

Dang LC, O'Neil JP and Jagust WJ (2012). Dopamine supports coupling of attentionrelated networks. J Neurosci 32, 9582-9587.

Derryberry D and Reed MA (1994). Temperament and attention: orienting toward and away from positive and negative signals. J Pers Soc Psychol 66, 1128-1139.

Diekhof EK, Kaps L, Falkai P and Gruber O (2012). The role of the human ventral striatum and the medial orbitofrontal cortex in the representation of reward magnitude an activation likelihood estimation meta-analysis of neuroimaging studies of passive reward expectancy and outcome processing. Neuropsychologia 50, 1252-66.

Engelmann JB, Damaraju E, Padmala S and Pessoa L (2009). Combined effects of attention and motivation on visual task performance: transient and sustained motivational effects. Front Hum Neurosci 3, 4.

Erhardt EB, Rachakonda S, Bedrick EJ Allen EA, Adali T and Calhoun VD (2011). Comparison of multi-subject ICA methods for analysis of fMRI data. Hum Brain Mapp 32, 2075-2095.

Ernst M, Pine DS and Hardin M (2006). Triadic model of the neurobiology of motivated behavior in adolescence. Psychol Med 36, 299-312.

Fischer H, Nyberg L, Karlsson S, Karlsson P, Brehmer Y, Rieckmann A, MacDonald SWS, Farde L and Bäckman L (2010). Simulating neurocognitive aging: effects of a dopaminergic antagonist on brain activity during working memory. Biol Psychiatry 67, $575-80$.

Friston KJ, Holmes AP, Worsley KJ, Poline JP, Frith CD and Frackowiak RSJ (1995). Statistical parametric maps in functional imaging: A general linear approach. Human Brain Mapping 2, 189-210. 
Greicius MD, Krasnow B, Reiss AL and Menon V (2003). Functional connectivity in the resting brain: a network analysis of the default mode hypothesis. Proc Natl Acad Sci U S A 100, 253-258.

Guitart-Masip M, Fuentemilla L, Bach DR, Huys QJM, Dayan P, Dolan RJ and Duzel E (2011). Action dominates valence in anticipatory representations in the human striatum and dopaminergic midbrain. J Neurosci 31, 7867-7875.

Haber SN and Knutson B (2010). The reward circuit: linking primate anatomy and human imaging. Neuropsychopharmacology 35, 4-26.

Hahn T, Dresler T, Ehlis AC, Plichta MM, Heinzel S, Polak T, Lesch KP, Breuer F, Jakob PM and Fallgatter AJ (2009). Neural response to reward anticipation is modulated by Gray's impulsivity. Neuroimage 46, 1148-1153.

Harrison BJ, Pujol J, Contreras-Rodríguez O, Soriano-Mas C, López-Solà M, Deus J, Ortiz H, Blanco-Hinojo L, Alonso P, Hernández-Ribas R, et al. (2011). Task-induced deactivation from rest extends beyond the default mode brain network. PLoS One 6, e22964.

Himberg J, Hyvärinen A and Esposito F (2004). Validating the independent components of neuroimaging time series via clustering and visualization. Neuroimage 22, 12141222.

Ivanov I, Liu X, Clerkin S, Schulz K, Friston K, Newcorn JH and Fan J (2012). Effects of motivation on reward and attentional networks: an fMRI study. Brain and Behavior $2,741-753$.

Jimura K, Locke HS and Braver TS (2010). Prefrontal cortex mediation of cognitive enhancement in rewarding motivational contexts. Proc Natl Acad Sci U S A 107, 88718876.

Juárez M, Kiehl KA, and Calhoun VD (2012). Intrinsic limbic and paralimbic networks are associated with criminal psychopathy. Hum Brain Mapp 00, 1-10.

Kim D, Manoach DS, Mathalon DH, Turner JA, Mannell M, Brown GG, Ford JM, Gollub RL, White T, Wible C, et al. (2009). Dysregulation of working memory and default-mode networks in schizophrenia using independent component analysis, an fBIRN and MCIC study. Hum Brain Mapp 30, 3795-3811.

Knutson B and Cooper JC (2005). Functional magnetic resonance imaging of reward prediction. Curr Opin Neurol 18, 411-417.

Knutson B, Adams CM, Fong GW and Hommer D (2001). Anticipation of increasing monetary reward selectively recruits nucleus accumbens. J Neurosci 21, RC159.

Knutson B and Greer SM (2008). Anticipatory affect: neural correlates and consequences for choice. Philos Trans R Soc Lond B Biol Sci 363, 3771-86. 
Krebs RM, Boehler CN, Roberts KC, Song AW and Woldorff MG (2012). The involvement of the dopaminergic midbrain and cortico-striatal-thalamic circuits in the integration of reward prospect and attentional task demands. Cereb Cortex 22, 607-615.

Kroger JK, Sabb FW, Fales CL, Bookheimer SY, Cohen MS and Holyoak KJ (2002). Recruitment of anterior dorsolateral prefrontal cortex in human reasoning: a parametric study of relational complexity. Cereb Cortex 12, 477-485.

Kruglanski A, Shah J, Fishbach A, Friedman R, Chun WY and Sleeth-Kepple D. (2002). A theory of goal systems. Adv. Exp. Soc. Psychol. 34, 331-378.

Langner R and Eickhoff SB. (2013). Sustaining Attention to Simple Tasks: A MetaAnalytic Review of the Neural Mechanisms of Vigilant Attention. Psychol Bull 139, 870-900.

Li YO, Adali $\mathrm{T}$ and Calhoun VD (2007). Estimating the number of independent components for functional magnetic resonance imaging data. Hum Brain Mapp 28, $1251-1266$.

Liu X, Hairston J, Schrier M and Fan J (2011). Common and distinct networks underlying reward valence and processing stages: a meta-analysis of functional neuroimaging studies. Neurosci Biobehav Rev 35, 1219-36.

Locke HS and Braver TS (2008). Motivational influences on cognitive control: behavior, brain activation, and individual differences. Cogn Affect Behav Neurosci 8 , 99-112.

Luman M, Van Meel CS, Oosterlaan J and Geurts HM (2012). Reward and punishment sensitivity in children with ADHD: validating the Sensitivity to Punishment and Sensitivity to Reward Questionnaire for children (SPSRQ-C). J Abnorm Child Psychol 40, 145-157.

Mohanty A, Gitelman DR, Small DM and Mesulam MM (2008). The spatial attention network interacts with limbic and monoaminergic systems to modulate motivationinduced attention shifts. Cereb Cortex 18, 2604-2613.

Nagano-Saito A, Liu J, Doyon J and Dagher A (2009). Dopamine modulates default mode network deactivation in elderly individuals during the Tower of London task. Neurosci Lett 458, 1-5.

Padmala S and Pessoa L (2011). Reward reduces conflict by enhancing attentional control and biasing visual cortical processing. J Cogn Neurosci 23, 3419-3432.

Parvaz MA, Konova AB, Tomasi D, Volkow ND and Goldstein RZ (2012). Structural integrity of the prefrontal cortex modulates electrocortical sensitivity to reward. J Cogn Neurosci 24, 1560-1570.

Patterson CM and Newman JP (1993). Reflectivity and learning from aversive events: toward a psychological mechanism for the syndromes of disinhibition. Psychol Rev 100, 716-736. 
Patterson CM, Kosson DS and Newman JP (1987). Reaction to punishment, reflectivity, and passive avoidance learning in extraverts. J Pers Soc Psychol 52, 565-575.

Pessoa L (2009). How do emotion and motivation direct executive control? Trends Cogn Sci 13, 160-166.

Petersen SE and Posner MI (2012). The attention system of the human brain: 20 years after. Annu Rev Neurosci 35, 73-89.

Pickering AD and Gray JA (2001). Dopamine, appetitive reinforcement, and the neuropsychology of human learning: An individual differences approach. In A. Eliasz, and A. Angleitner, eds. Advances in Research on Temperament, (Lengerich, Germany: PABST Science Publishers), pp. 113-149.

Ptak R and Schnider A (2010). The dorsal attention network mediates orienting toward behaviorally relevant stimuli in spatial neglect. J Neurosci 30, 12557-12565.

Raichle ME and Snyder AZ (2007). A default mode of brain function: a brief history of an evolving idea. Neuroimage 37, 1083-90; discussion 1097-9.

Raichle ME, MacLeod AM, Snyder AZ, Powers WJ, Gusnard DA and Shulman GL (2001). A default mode of brain function. Proc Natl Acad Sci U S A 98, 676-682.

Sambataro F, Blasi G, Fazio L, Caforio G, Taurisano P, Romano R, Di Giorgio A, Gelao B, Lo Bianco L, Papazacharias A, et al. (2010). Treatment with olanzapine is associated with modulation of the default mode network in patients with Schizophrenia. Neuropsychopharmacology 35, 904-912.

Schultz W, Tremblay L and Hollerman JR (1998). Reward prediction in primate basal ganglia and frontal cortex. Neuropharmacology 37, 421-429.

Segall JM, Allen EA, Jung RE, Erhardt EB, Arja SK, Kiehl K, and Calhoun VD (2012). Correspondence between structure and function in the human brain at rest. Front Neuroinformatics 6,10 .

Sescousse G, Caldú X, Segura B and Dreher JC (2013). Processing of primary and secondary rewards: a quantitative meta-analysis and review of human functional neuroimaging studies. Neurosci Biobehav Rev 37, 681-96.

Small DM, Gitelman D, Simmons K, Bloise SM, Parrish T and Mesulam MM (2005). Monetary incentives enhance processing in brain regions mediating top-down control of attention. Cereb Cortex 15, 1855-1865.

Spitzer H, Desimone R and Moran J (1988). Increased attention enhances both behavioral and neuronal performance. Science 240, 338-40.

Spreng RN (2012). The fallacy of a "task-negative" network. Frontiers in Psychology 3, 145 . 
Spreng RN, Stevens WD, Chamberlain JP, Gilmore AW and Schacter DL (2010). Default network activity, coupled with the frontoparietal control network, supports goal-directed cognition. Neuroimage 53, 303-317.

Stalnaker TA, Calhoon GG, Ogawa M, Roesch MR and Schoenbaum G (2012). Reward prediction error signaling in posterior dorsomedial striatum is action specific. $\mathbf{J}$ Neurosci 32, 10296-10305.

Tan HY, Chen Q, Goldberg TE, Mattay VS, Meyer-Lindenberg A, Weinberger DR and Callicott JH (2007). Catechol-O-methyltransferase Val158Met modulation of prefrontal-parietal-striatal brain systems during arithmetic and temporal transformations in working memory. J Neurosci 27, 13393-13401.

Tomasi D, Volkow ND, Wang GJ, Wang R, Telang F, Caparelli EC, Wong C, Jayne M and Fowler JS (2011). Methylphenidate enhances brain activation and deactivation responses to visual attention and working memory tasks in healthy controls. Neuroimage 54, 3101-3110.

Torrubia R, Avila C, Molto J and Caseras X (2001). The Sensitivity to Punishment and Sensitivity to Reward Questionnaire (SPSRQ) as a measure of Gray's anxiety and impulsivity dimensions. Pers Individ Dif 31, 837-862.

Van den Heuvel OA, Groenewegen HJ, Barkhof F, Lazeron RHC, Van Dyck R and Veltman DJ (2003). Frontostriatal system in planning complexity: a parametric functional magnetic resonance version of Tower of London task. Neuroimage 18, 367374.

Van Lankveld JJDM and Smulders FTY (2008). The effect of visual sexual content on the event-related potential. Biol Psychol 79, 200-8.

Vincent JL, Kahn I, Snyder AZ, Raichle ME and Buckner RL (2008). Evidence for a frontoparietal control system revealed by intrinsic functional connectivity. $\mathrm{J}$ Neurophysiol 100, 3328-3342.

Volkow ND, Wang GJ, Fowler JS, Tomasi D, and Telang F (2011). Addiction: beyond dopamine reward circuitry. Proc Natl Acad Sci U S A 108, 15037-15042.

Wager TD, Jonides J and Reading S (2004). Neuroimaging studies of shifting attention: a meta-analysis. Neuroimage 22, 1679-1693.

Wightman RM and Robinson DL (2002). Transient changes in mesolimbic dopamine and their association with "reward". J Neurochem 82, 721-735.

Williams-Gray CH, Hampshire A, Robbins TW, Owen AM and Barker RA (2007). Catechol O-methyltransferase Val158Met genotype influences frontoparietal activity during planning in patients with Parkinson's disease. J Neurosci 27, 4832-4838.

Xu J, Potenza MN and Calhoun VD (2013) Spatial ICA reveals functional activity hidden from traditional fMRI GLM-based analyses. Front. Neurosci. 7, 154. 
Ye Z, Doñamayor N and Münte TF (2012). Brain network of semantic integration in sentence reading: Insights from independent component analysis and graph theoretical analysis. Hum Brain Mapp 000.

Yeung N and Sanfey AG (2004). Independent coding of reward magnitude and valence in the human brain. J Neurosci 24, 6258-64.

Table 1: Behavioral data

\begin{tabular}{|c|c|c|c|c|}
\hline \multicolumn{5}{|c|}{ Experiment 1} \\
\hline \multirow[t]{2}{*}{ Behavioral } & \multicolumn{2}{|c|}{ Reaction Time (ms) } & \multicolumn{2}{|c|}{ Hits $(\%)$} \\
\hline & $M$ & $S D$ & $M$ & $S D$ \\
\hline Reward & 187.7 & 28.3 & 84.2 & 10.8 \\
\hline Punishment & 190.1 & 30.5 & 80.3 & 10.2 \\
\hline \multicolumn{5}{|c|}{ Experiment 2} \\
\hline \multirow[t]{2}{*}{ Behavioral } & \multicolumn{2}{|c|}{ Reaction Time (ms) } & \multicolumn{2}{|c|}{ Hits (\%) } \\
\hline & $M$ & $S D$ & $M$ & $S D$ \\
\hline $\mathrm{CR}$ & 235.9 & 57.7 & - & - \\
\hline PR & 217.8 & 58.2 & 59.7 & 7.4 \\
\hline $\mathrm{N}$ & 245.6 & 65.7 & - & - \\
\hline \multirow[t]{2}{*}{ Image ratings } & \multicolumn{2}{|c|}{ Valence } & \multicolumn{2}{|c|}{ Arousal } \\
\hline & $M$ & $S D$ & $M$ & $S D$ \\
\hline Appetitive set & 6.8 & 1.1 & 6.4 & 1.3 \\
\hline Neutral set & 4.7 & 1 & 3.4 & 1.2 \\
\hline
\end{tabular}

$\mathrm{M}=$ mean; $\mathrm{SD}=$ standard deviation; $\mathrm{ms}=$ milliseconds; $\mathrm{CR}=$ Continuous reward; $\mathrm{PR}=$ Partial reward; $\mathrm{N}=\mathrm{Neutral}$ 
Table 2 Brain regions belonging the ICs of interest

\begin{tabular}{|c|c|c|c|c|c|}
\hline Component & Region & $\begin{array}{l}\text { Brodmann } \\
\text { areas }\end{array}$ & $\begin{array}{c}\text { Max. MNI } \\
\text { coordinates }\end{array}$ & $k$ & $\begin{array}{l}\text { Max. } t \\
\text { value }\end{array}$ \\
\hline \multicolumn{6}{|l|}{ Experiment 1} \\
\hline \multicolumn{6}{|l|}{$\begin{array}{l}\text { Default Mode } \\
\text { Network (CO4) }\end{array}$} \\
\hline & Precuneus & $\begin{array}{c}7,30,29 \\
31\end{array}$ & $-9-5528$ & 1754 & 34.43 \\
\hline & Medial PFC & $\begin{array}{c}10,32,9 \\
11\end{array}$ & -6537 & 693 & 23.75 \\
\hline & Talamus & - & $-6-137$ & 62 & 14.91 \\
\hline & Angular gyrus left & 39 & $-42-7034$ & 73 & 14.28 \\
\hline & Angular gyrus right & 39 & $42-6737$ & 32 & 13.53 \\
\hline \multicolumn{6}{|l|}{$\begin{array}{c}\text { Left } \\
\text { Frontoparietal } \\
\text { Network (C05) }\end{array}$} \\
\hline & Inferior parietal left & $\begin{array}{c}40,39,21 \\
22,31,7 \\
19\end{array}$ & $-51-5243$ & 2432 & 25.83 \\
\hline & Superior frontal cortex & 8,6 & -123552 & 767 & 19.46 \\
\hline & $\begin{array}{l}\text { Inferior frontal cortex } \\
\text { left }\end{array}$ & 47,45 & $-5129-5$ & 256 & 16.40 \\
\hline & Inferior parietal right & 40 & $54-6140$ & 133 & 15.41 \\
\hline & Postcentralgyrus left & 4 & $-21-3758$ & 49 & 13.08 \\
\hline \multicolumn{6}{|l|}{$\begin{array}{c}\text { Right } \\
\text { Frontoparietal } \\
\text { Network (CO9) }\end{array}$} \\
\hline & Inferior parietal right & $40,7,39$ & $54-5843$ & 1064 & 24.62 \\
\hline & Inferior parietal left & 40,7 & $-39-5846$ & 453 & 19.73 \\
\hline & $\begin{array}{l}\text { Middle frontal gyrus } \\
\text { right }\end{array}$ & $\begin{array}{c}8,9,10,6 \\
46\end{array}$ & 451449 & 878 & 19.18 \\
\hline & Middle Temporal right & 21 & $63-34-8$ & 143 & 16.69 \\
\hline & Medial PFC & 8 & 03246 & 152 & 16.07 \\
\hline & Posterior cingulate & 31 & $3-3128$ & 95 & 15.85 \\
\hline & Precuneus & 7 & $3-7646$ & 40 & 12.68 \\
\hline \multicolumn{6}{|l|}{$\begin{array}{c}\text { Frontal } \\
\text { network (C10) }\end{array}$} \\
\hline & Medial and lateral PFC & $\begin{array}{c}10,8,9,32 \\
6,24,11\end{array}$ & -3504 & 4862 & 28.1 \\
\hline & $\begin{array}{l}\text { Inferior frontal cortex } \\
\text { left }\end{array}$ & 47,45 & $-4514-5$ & 252 & 19.81 \\
\hline \multicolumn{6}{|l|}{ Experiment 2} \\
\hline \multicolumn{6}{|l|}{$\begin{array}{l}\text { Sensory motor } \\
\text { network ( } \mathrm{CO} 3)\end{array}$} \\
\hline & Poscentralgyrus left & $\begin{array}{c}3,40,2,4 \\
6\end{array}$ & $-42-3155$ & 595 & 24.8 \\
\hline \multicolumn{6}{|l|}{$\begin{array}{c}\text { Right } \\
\text { Frontoparietal } \\
\text { Network } \\
\text { (CO7) }\end{array}$} \\
\hline & $\begin{array}{l}\text { Middle frontal gyrus } \\
\text { right }\end{array}$ & $10,8,9$ & $3653-2$ & 822 & 26.49 \\
\hline & Inferior parietal right & 40,7 & $39-58 \quad 43$ & 576 & 26.17 \\
\hline & Inferior parietal left & 40 & $-45-5249$ & 159 & 24.01 \\
\hline & Posterior cingulate & 31 & $3-2837$ & 64 & 17.79 \\
\hline \multicolumn{6}{|l|}{$\begin{array}{l}\text { Default Mode } \\
\text { Network } \\
\text { (C19) }\end{array}$} \\
\hline & Angular gyrus left & 19,39 & $-42-82 \quad 31$ & 170 & 28.43 \\
\hline & Precuneus & 29,30 & $9-5516$ & 323 & 21.48 \\
\hline & Medial PFC & 10, 11 & $044-14$ & 146 & 19.33 \\
\hline & Angular gyrus right & 39 & $42-82 \quad 34$ & 146 & 18.67 \\
\hline \multicolumn{6}{|l|}{$\begin{array}{l}\text { Cerebellum } \\
\text { network (C26) }\end{array}$} \\
\hline & Cerebellum & - & $-30-46-35$ & 1635 & 34.84 \\
\hline
\end{tabular}


Table 3 Pearson correlations between the SR scores and IC task-related modulation

\begin{tabular}{|c|c|c|c|c|}
\hline \multicolumn{5}{|c|}{ Experiment 1} \\
\hline Condition & DMN (C04) & $\begin{array}{l}\text { Left FPN } \\
(\mathrm{CO5})\end{array}$ & $\begin{array}{l}\text { Right FPN } \\
\text { (C09) }\end{array}$ & $\begin{array}{c}\text { Frontal } \\
\text { network } \\
\text { (C10) }\end{array}$ \\
\hline Reward & $-0.41 * *$ & 0.01 & $0.32 *$ & 0.01 \\
\hline Punishment & $-0.45^{* *}$ & -0.04 & $0.35^{*}$ & -0.01 \\
\hline Neutral & -0.16 & -0.16 & -0.04 & 0.03 \\
\hline \multicolumn{5}{|c|}{ Experiment 2} \\
\hline Condition & $\begin{array}{c}\text { Sensory } \\
\text { motor } \\
\text { network } \\
\text { (CO3) }\end{array}$ & $\begin{array}{l}\text { Right FPN } \\
\text { (CO7) }\end{array}$ & DMN (C19) & $\begin{array}{l}\text { Cerebellum } \\
\text { network } \\
\text { (C26) }\end{array}$ \\
\hline $\begin{array}{c}\text { Continuous } \\
\text { reward }\end{array}$ & 0.17 & $0.63^{* *}$ & $-0.48 * *$ & -0.03 \\
\hline Partial reward & 0.11 & $0.52 * *$ & $-0.45^{*}$ & -0.02 \\
\hline Neutral & 0.11 & $0.49 * *$ & -0.07 & -0.21 \\
\hline Control & 0.05 & $0.42 *$ & -0.19 & -0.10 \\
\hline
\end{tabular}

Fig. 1 Activity in VS ROIs obtained from GLM analyses. Images are presented in neurological convention (left is left) and with a threshold at $\mathrm{p}<0.05$ FWE corrected. The color bar represents the $t$ values applicable to the image.

Fig. 2 Mean and standard error bars for the anticipatory cues beta-weights in each component (C) of interest. Experiment 1: C04, default mode network; C05, left frontoparietal network; C09, right frontoparietal network; C10, frontal network. Experiment 2: C03, sensory motor network; C07, right frontoparietal network; C19, default mode network; C26, cerebellum network.

* Significant differences at $\mathrm{p}<0.05$ corrected.

Fig. 3 Networks showing task-related modulation in both experiments with their associated event-related averages. Images are presented in neurological convention (left is left). The statistical threshold is $\mathrm{p}<1 \times 10^{-12}$ FDR-corrected with a minimum extent threshold of 30 contiguous voxels. The color bar represents the t values applicable to the image, while the numbers in the images correspond to the $\mathrm{z}$ MNI coordinates. 


\section{Experiment 1}

C04 DMN

(2)

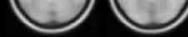

(i)

8

(1)

C

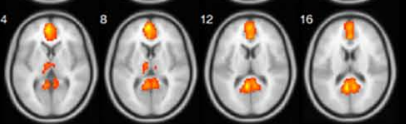

"A " (3)

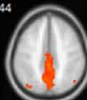

(1)
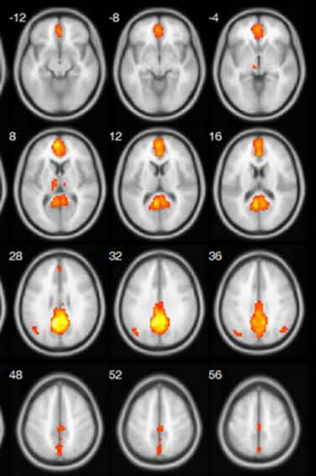

(1)

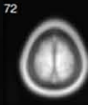

C09 right FPN
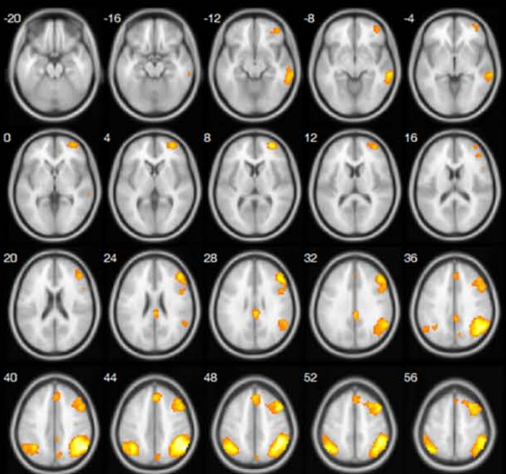

(2)
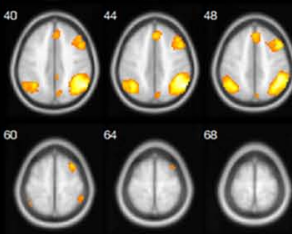

(1)
(ㅇ)

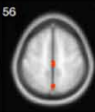

Experiment 2
C19 DMN

(2)

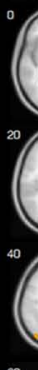

(3)

(1)
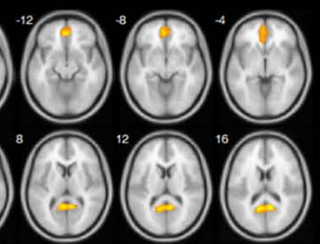

$\Delta 0000$

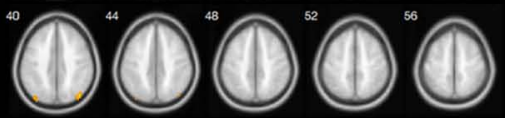

\section{(1)}

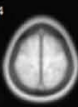

C07 right FPN
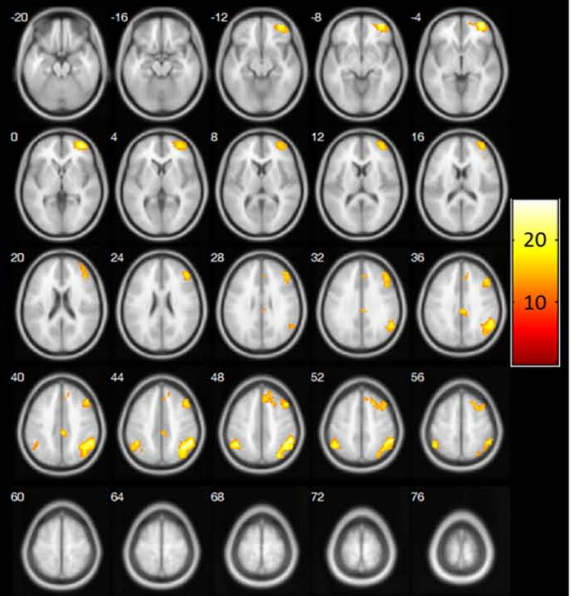

C04

- Reward

- - Punishment

....... Non-Incentive
23456789101112131415

Time (seconds)

C19

$$
0,1
$$

0,05

๖ั้

-

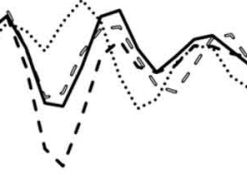

$-0,2$

123456789101112131415 Time (seconds)

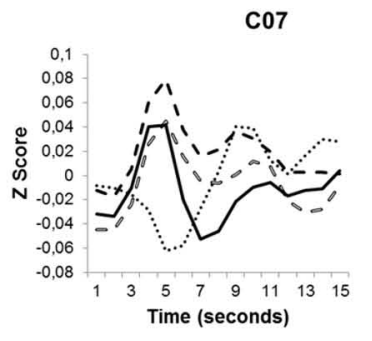

Reward

- - Punishment

..... Non-Incentive

- Continuous Reward

- - Partial

Reward

- - Neutral

....... Control<smiles>C1CCC2CCCC2C1</smiles> 


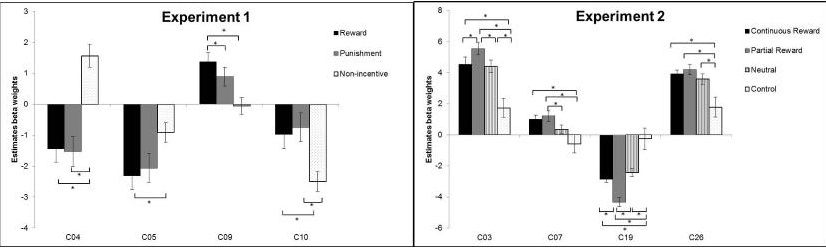




\section{Experiment 1}

Reward > Non-incentive

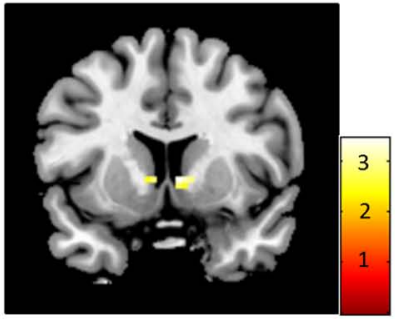

Punishment $>$ Non-incentive

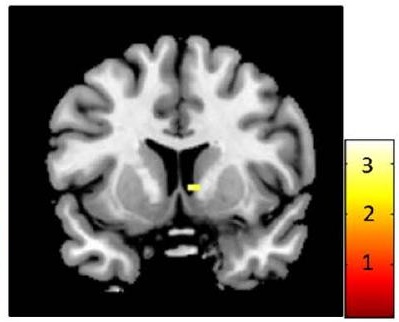

\section{Experiment 2}

Continuous reward $>$ Neutral

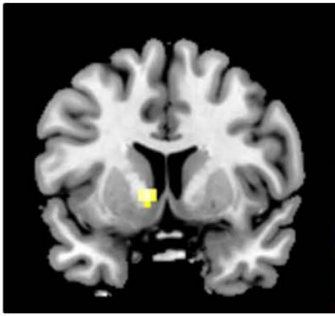

Partial reward $>$ Neutral

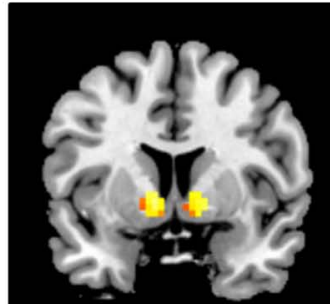

Continuous reward $>$ Control
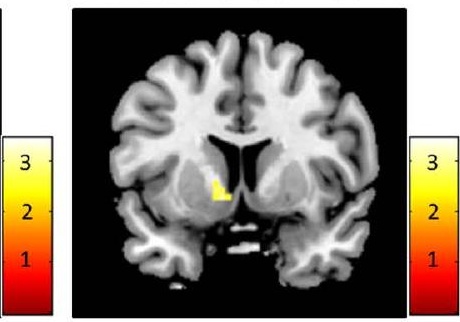

Partial reward $>$ Control

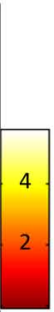

